\title{
ON AN ANTIPLANE CRACK PROBLEM FOR FUNCTIONALLY GRADED ELASTIC MATERIALS
}

\author{
DAVID L. CLEMENTS ${ }^{1}$
}

(Received 20 October, 2009; revised 10 November, 2010)

\begin{abstract}
This paper examines an antiplane crack problem for a functionally graded anisotropic elastic material in which the elastic moduli vary quadratically with the spatial coordinates. A solution to the crack problem is obtained in terms of a pair of integral equations. An iterative solution to the integral equations is used to examine the effect of the anisotropy and varying elastic moduli on the crack tip stress intensity factors and the crack displacement.
\end{abstract}

2000 Mathematics subject classification: primary 35J25; secondary 74B05, 74E10. Keywords and phrases: crack problems, anisotropy, functionally graded materials.

\section{Introduction}

In recent years there has been an increasing interest in the class of inhomogeneous materials that are now commonly called functionally graded materials (FGMs). Typically such materials are composites that may be characterized by gradual variation in the composition and volume fractions of the constituents that comprise the FGM (Paulino [19], Riedel et al. [21], Hassanin and Jiang [14] and Gelbstein et al. [13]). This gives rise to a material with a nonuniform microstructure and a continuously graded macrostructure. Thus from a macro viewpoint FGMs have elastic moduli with gradients that vary continuously with position. As a consequence, within the linear theory of elasticity they are modelled by linear partial differential equations, with the variable elastic moduli giving rise to variable coefficients with continuous gradients.

FGMs are employed in a variety of applications. They are used as coatings to enhance desired surface properties and to reduce the residual stress and thermal stress at the interface between the coating and the underlying material. They are also used as interfacial layers in order to reduce the stress arising from the material property discontinuities occurring, for example, in biomedical applications such as orthopaedic implants for hip and knee joint replacement (Pompe et al. [20]). Applications

\footnotetext{
${ }^{1}$ School of Mathematics, University of Adelaide, Adelaide, SA 5005, Australia; e-mail: david.clements@ adelaide.edu.au.

(C) Australian Mathematical Society 2011, Serial-fee code 1446-1811/2011 \$16.00
} 
involving FGMs comprising metal-ceramic combinations are advantageously employed in coatings or interfacial layers to reduce thermal stresses in layered materials in severe temperature environments (Zhang et al. [23], Noda [17]).

In these applications, consideration of the fracture of FGMs is of importance. In this context, a number of authors have considered crack problems in FGMs. Some of the earlier studies include the work of Clements et al. [8] and Erdogan [10] while other examples include papers by Erdogan and Ozturk [11], Konda and Erdogan [16], Chen and Erdogan [4] and Jin and Batra [15]. More recent studies include papers by Chan et al. [3], Dag and Erdogan [9], Noda and Wang [18], Bohr [2], Clements and Ang [7] and Chen et al. [5].

The current study is concerned with an antiplane crack problem for FGMs under antiplane shear loading. Expressions for the antiplane displacement and stress for a wide class of these materials are obtained in terms of a single analytic function of a complex variable. These representations are used to consider a crack problem for an FGM in which the elastic moduli exhibit quadratic variation with the spatial Cartesian coordinates. The problem is solved in terms of integral equations which yield iterative solutions for a limited range of the elastic moduli. Equations are obtained for the crack tip stress intensity factors and crack displacement. These equations yield information regarding the effect of the varying elastic moduli and anisotropy on the stress intensity factors and the crack displacement.

Numerical results are presented for a particular anisotropic material in order to provide a quantitative illustration of the use of an FGM to reduce crack tip stress intensity factors and the crack displacement.

\section{Statement of the problem}

Consider an anisotropic functionally graded elastic body which, referred to a Cartesian frame $O x_{1} x_{2} x_{3}$, has a geometry and elastic moduli $c_{i j k l}\left(x_{1}, x_{2}\right)$ that do not vary in the $O x_{3}$ direction. The anisotropic material exhibits elastic symmetry with respect to the coordinate plane $x_{3}=0$. The material contains a crack in the region $\left|x_{1}\right|<a$ on $x_{2}=0$ and is otherwise continuous throughout the whole space $R^{3}$ (Figure 1). Over the crack faces the only nonzero stress is the specified antiplane stress which does not vary in the $O x_{3}$ direction. The problem is to determine the displacement and stress throughout the material and, in particular, to obtain the crack tip stress intensity factors and the displacement over the crack faces.

In view of the geometry, the elastic symmetry and the boundary conditions, it is appropriate to seek a solution to this problem which only involves the two Cartesian coordinates $x_{1}$ and $x_{2}$, the antiplane displacement $u_{3}$ and the antiplane stresses $\sigma_{13}$ and $\sigma_{23}$.

\section{Basic equations}

The equilibrium equation governing small antiplane deformations of a functionally graded elastic material that exhibits elastic symmetry with respect to the coordinate 


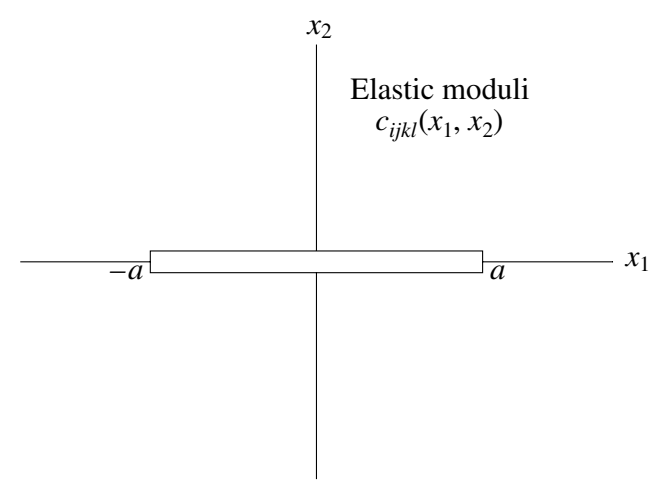

FIGURE 1. Crack geometry.

plane $x_{3}=0$ is given by

$$
\frac{\partial}{\partial x_{j}}\left[c_{3 j 3 l}(\mathbf{x}) \frac{\partial u_{3}(\mathbf{x})}{\partial x_{l}}\right]=0,
$$

where $k, l=1,2, \mathbf{x}=\left(x_{1}, x_{2}\right), u_{3}$ is the antiplane displacement, $c_{3 j 3 l}(\mathbf{x})$ for $i, j=$ 1,2 are the relevant elastic moduli and the repeated summation convention (summing from 1 to 2) is used for repeated Latin suffices. The stress displacement relations are

$$
\sigma_{3 j}(\mathbf{x})=c_{3 j 3 l} \frac{\partial u_{3}}{\partial x_{l}}
$$

for $j=1,2$. The antiplane component of the stress vector on a boundary with outward pointing normal $\mathbf{n}=\left(n_{1}, n_{2}\right)$ is given by

$$
P(\mathbf{x})=\sigma_{3 j} n_{j}=c_{3 j} 3 l \frac{\partial u_{3}}{\partial x_{l}} n_{j} .
$$

For all points in the elastic body, the coefficients $c_{3 j 3 l}(\mathbf{x})$ satisfy the usual symmetry conditions

$$
c_{3 j 3 l}=c_{3 j l 3}=c_{j 33 l}=c_{3 l 3 j} .
$$

Also, in order to satisfy the condition that the strain energy density be positive, the coefficients must satisfy the inequality

$$
c_{3232} c_{3131}-c_{3231}^{2}>0 \text {. }
$$

The variation in the coefficients in (3.1) is restricted to take the form

$$
c_{3 j 3 l}(\mathbf{x})=c_{3 j 3 l}^{(0)} g(\mathbf{x}),
$$

where the $c_{3 j 3 l}^{(0)}$ are constants and $g\left(x_{1}, x_{2}\right)>0$ is a twice-differentiable function of the variables $x_{1}$ and $x_{2}$. Use of (3.4) in (3.1) yields

$$
c_{3 j 3 l}^{(0)} \frac{\partial}{\partial x_{j}}\left(g \frac{\partial u_{3}}{\partial x_{l}}\right)=0 .
$$


Following Azis and Clements [1], a new dependent variable is introduced by the transformation

$$
u_{3}=g^{-1 / 2} \psi
$$

Substituting (3.6) in (3.5), it follows that (3.5) will be satisfied if $g$ and $\psi$ are solutions of the equations

$$
c_{3 j 3 l}^{(0)} \frac{\partial^{2} \psi}{\partial x_{j} \partial x_{l}}=0
$$

and

$$
c_{3 j 3 l}^{(0)} \frac{\partial^{2} g^{1 / 2}}{\partial x_{j} \partial x_{l}}=0 .
$$

The general solution of (3.7) and (3.8) may be written in terms of an analytic function in the form (Eshelby et al. [12], Clements [6])

$$
\begin{aligned}
g^{1 / 2} & =2 \Re\left[f\left(z_{\alpha}\right)\right], \\
\psi & =2 \Re\left[\theta\left(z_{\alpha}\right)\right],
\end{aligned}
$$

where $\mathfrak{R}$ denotes the real part of a complex number and $f\left(z_{\alpha}\right)$ and $\theta\left(z_{\alpha}\right)$ are arbitrary analytic functions of the complex variable $z_{\alpha}=x_{1}+\tau x_{2}$, with $\tau$ the root with positive imaginary part of the quadratic

$$
c_{3131}^{(0)}+\left(c_{3231}^{(0)}+c_{3132}^{(0)}\right) \tau+c_{3232}^{(0)} \tau^{2}=0 .
$$

A solution to (3.8) which is applicable to the crack problem in Section 2 takes the form $g(\mathbf{x})=\left(\beta x_{2}+\gamma\right)^{2}$, where $\beta$ and $\gamma$ are constants.

From (3.2), (3.6) and (3.9), expressions for the displacement and stress may be written in the form

$$
\begin{gathered}
u_{3}=2 g^{-1 / 2} \Re\left[\theta\left(z_{\alpha}\right)\right], \\
\sigma_{3 j}=2 \Re\left[-c_{3 j 3 l}^{(0)} \frac{\partial g^{1 / 2}}{\partial x_{l}} \theta\left(z_{\alpha}\right)+g^{1 / 2} L_{3 j} \theta^{\prime}\left(z_{\alpha}\right)\right],
\end{gathered}
$$

where primes denote differentiation with respect to the argument in question, and

$$
L_{3 j}=c_{3 j 31}^{(0)}+\tau_{\alpha} c_{3 j 32}^{(0)} \text {. }
$$

From (3.10) and (3.3) it follows that

$$
\tau_{\alpha}=\frac{-c_{3232}^{(0)}+i\left[c_{3232}^{(0)} c_{3131}^{(0)}-\left(c_{3231}^{(0)}\right)^{2}\right]^{1 / 2}}{c_{3232}^{(0)}}
$$

and hence (3.13) yields

$$
L_{32}=i\left[c_{3232}^{(0)} c_{3131}^{(0)}-\left(c_{3231}^{(0)}\right)^{2}\right]^{1 / 2},
$$

showing that $L_{32}$ has zero real part. 
On $x_{2}=0,(3.11)$ and (3.12) yield

$$
\begin{gathered}
u_{3}=g^{-1 / 2}\left[\theta\left(x_{1}\right)+\bar{\theta}\left(x_{1}\right)\right], \\
\sigma_{32}=-c_{323 l}^{(0)} \frac{\partial g^{1 / 2}}{\partial x_{l}}\left[\theta\left(x_{1}\right)+\bar{\theta}\left(x_{1}\right)\right]+g^{1 / 2}\left[L_{32} \theta^{\prime}\left(x_{1}\right)+\bar{L}_{32} \bar{\theta}^{\prime}\left(x_{1}\right)\right],
\end{gathered}
$$

where the bar denotes the complex conjugate.

An alternative representation for the displacement and stress may be obtained by putting

$$
L_{32} \theta(z)=\chi(z)
$$

where $\chi(z)$ is an analytic function of the complex variable $z$. The constant $L_{32}$ is nonzero (see Clements [6], Stroh [22]), and hence from (3.15),

$$
\theta(z)=M \chi(z),
$$

where

$$
M=L_{32}^{-1} \text {. }
$$

Substitution of (3.16) into (3.11) and (3.12) yields

$$
\begin{gathered}
u_{3}=2 g^{-1 / 2} \Re\left[M \chi\left(z_{\alpha}\right)\right], \\
\sigma_{3 j}=2 \Re\left[-c_{3 j 3 l}^{(0)} \frac{\partial g^{1 / 2}}{\partial x_{l}} M \chi\left(z_{\alpha}\right)+g^{1 / 2} L_{3 j} M \chi^{\prime}\left(z_{\alpha}\right)\right] .
\end{gathered}
$$

In particular, on $x_{2}=0,(3.18)$ and (3.19) yield

$$
\begin{gathered}
u_{3}=g^{-1 / 2}\left[M \chi\left(x_{1}\right)+\bar{M} \bar{\chi}\left(x_{1}\right)\right], \\
\sigma_{32}=-c_{323 l}^{(0)} \frac{\partial g^{1 / 2}}{\partial x_{l}}\left[M \chi\left(x_{1}\right)+\bar{M} \bar{\chi}\left(x_{1}\right)\right]-g^{1 / 2}\left[\chi^{\prime}\left(x_{1}\right)+\bar{\chi}^{\prime}\left(x_{1}\right)\right] .
\end{gathered}
$$

\section{A crack in a material with modulus $c_{i j k l}=c_{i j k l}^{(0)}\left(\beta\left|x_{2}\right|+\gamma\right)^{2}$}

Consider an inhomogeneous elastic material with elastic modulus given by

$$
c_{i j k l}=c_{i j k l}^{(0)}\left(\beta\left|x_{2}\right|+\gamma\right)^{2},
$$

where the constants $\beta$ and $\gamma$ satisfy the inequalities $\beta \geq 0$ and $\gamma>0$. The material contains a crack along $x_{2}=0$ for $\left|x_{1}\right|<a$, where $a$ is a positive constant. Over the crack faces the stress vector $P$ is prescribed. The displacement and stress fields are required throughout the material. For this problem the representation (3.18)-(3.21) is useful with $\chi(z)$ given by

$$
\chi(z)= \begin{cases}\frac{1}{2 \pi} \int_{0}^{\infty} G^{+}(p) \exp (i p z) d p & \text { for } x_{2}>0 \\ \frac{1}{2 \pi} \int_{0}^{\infty} G^{-}(p) \exp (-i p z) d p & \text { for } x_{2}<0,\end{cases}
$$


where $G^{+}(p)$ and $G^{-}(p)$ are functions of $p$ which will be determined by the boundary conditions. From (3.18) and (4.2) it follows that

$$
u_{3}=\frac{g^{-1 / 2}}{2 \pi}\left[M \int_{0}^{\infty} G^{+}(p) \exp \left(i p z_{\alpha}\right) d p+\bar{M} \int_{0}^{\infty} \bar{G}^{+}(p) \exp \left(-i p \bar{z}_{\alpha}\right) d p\right]
$$

for $x_{2}>0$, and

$$
u_{3}=\frac{g^{-1 / 2}}{2 \pi}\left[M \int_{0}^{\infty} G^{-}(p) \exp \left(-i p z_{\alpha}\right) d p+\bar{M} \int_{0}^{\infty} \bar{G}^{-}(p) \exp \left(i p \bar{z}_{\alpha}\right) d p\right]
$$

for $x_{2}<0$. Equations (3.4), (4.1) and (3.19) yield the antiplane stress in the form

$$
\begin{aligned}
\sigma_{3 j}=\frac{1}{2 \pi} & {\left[-c_{3 j 32}^{(0)} \beta M \int_{0}^{\infty} G^{+}(p) \exp \left(i p z_{\alpha}\right) d p\right.} \\
& +g^{1 / 2} L_{3 j} M \int_{0}^{\infty} G^{+}(p) \exp \left(i p z_{\alpha}\right) i p d p \\
& -c_{3 j 32}^{(0)} \beta \bar{M} \int_{0}^{\infty} \bar{G}^{+}(p) \exp \left(-i p \bar{z}_{\alpha}\right) d p \\
& \left.-g^{1 / 2} \bar{L}_{3 j} \bar{M} \int_{0}^{\infty} \bar{G}^{+}(p) \exp \left(-i p \bar{z}_{\alpha}\right) i p d p\right]
\end{aligned}
$$

for $x_{2}>0$, and

$$
\begin{aligned}
\sigma_{3 j}=\frac{1}{2 \pi} & {\left[c_{3 j 32}^{(0)} \beta M \int_{0}^{\infty} G^{-}(p) \exp \left(-i p z_{\alpha}\right) d p\right.} \\
& -g^{1 / 2} L_{3 j} M \int_{0}^{\infty} G^{-}(p) \exp \left(-i p z_{\alpha}\right) i p d p \\
& +c_{3 j 32}^{(0)} \beta \bar{M} \int_{0}^{\infty} \bar{G}^{-}(p) \exp \left(i p \bar{z}_{\alpha}\right) d p \\
& \left.+g^{1 / 2} \bar{L}_{3 j} \bar{M} \int_{0}^{\infty} \bar{G}^{-}(p) \exp \left(i p \bar{z}_{\alpha}\right)(i p) d p\right]
\end{aligned}
$$

for $x_{2}<0$.

Since $L_{32}$ has zero real part, it follows from (3.17) that $M$ has zero real part. Denoting the imaginary part of $M$ by $m$, the antiplane stress and displacement on the boundary $x_{2}=0$ may be written in the form

$$
\begin{aligned}
u_{3}\left(x_{1}, 0+\right)= & \frac{i m}{2 \pi \gamma}\left[\int_{0}^{\infty} G^{+}(p) \exp \left(i p x_{1}\right) d p\right. \\
& \left.-\int_{0}^{\infty} \bar{G}^{+}(p) \exp \left(-i p x_{1}\right) d p\right], \\
u_{3}\left(x_{1}, 0-\right)=\frac{i m}{2 \pi \gamma} & {\left[\int_{0}^{\infty} G^{-}(p) \exp \left(-i p x_{1}\right) d p\right.} \\
& \left.-\int_{0}^{\infty} \bar{G}^{-}(p) \exp \left(i p x_{1}\right) d p\right],
\end{aligned}
$$




$$
\begin{aligned}
\sigma_{32}\left(x_{1}, 0+\right)=\frac{1}{2 \pi} & {\left[-c_{3232}^{(0)} \beta i m \int_{0}^{\infty} G^{+}(p) \exp \left(i p x_{1}\right) d p\right.} \\
& +\gamma \int_{0}^{\infty} G^{+}(p) \exp \left(i p x_{1}\right) i p d p \\
& +c_{3232}^{(0)} \beta i m \int_{0}^{\infty} \bar{G}^{+}(p) \exp \left(-i p x_{1}\right) d p \\
& \left.-\gamma \int_{0}^{\infty} \bar{G}^{+}(p) \exp \left(-i p x_{1}\right) i p d p\right], \\
\sigma_{32}\left(x_{1}, 0-\right)=\frac{1}{2 \pi} & {\left[c_{3232}^{(0)} \beta i m \int_{0}^{\infty} G^{-}(p) \exp \left(-i p x_{1}\right) d p\right.} \\
& -\gamma \int_{0}^{\infty} G^{-}(p) \exp \left(-i p x_{1}\right) i p d p \\
& -c_{3232}^{(0)} \beta i m \int_{0}^{\infty} \bar{G}^{-}(p) \exp \left(i p x_{1}\right) d p \\
& \left.+\gamma \int_{0}^{\infty} \bar{G}^{-}(p) \exp \left(i p x_{1}\right) i p d p\right] .
\end{aligned}
$$

Define

$$
G^{+}=\bar{G}^{-}=G
$$

The difference in displacement across $x_{2}=0$ is

$$
\Delta u_{3}=\frac{g^{-1 / 2}}{\pi} \Re\left[2 i m \int_{0}^{\infty} G(p) \exp \left(i p x_{1}\right) d p\right] .
$$

In view of (4.7), it follows from (4.5) and (4.6) that the shear stress is continuous across $x_{2}=0$. Let $\sigma_{E}$ and $\sigma_{O}$ denote the even and odd parts of the stress $\sigma_{32}\left(x_{1}, 0\right)$, respectively, so that

$$
\sigma_{E}=\frac{\sigma_{32}\left(x_{1}, 0\right)+\sigma_{32}\left(-x_{1}, 0\right)}{2}, \quad \sigma_{O}=\frac{\sigma_{32}\left(x_{1}, 0\right)-\sigma_{32}\left(-x_{1}, 0\right)}{2} .
$$

Hence from (4.5), (4.7) and (4.9), it follows that on $x_{2}=0$,

$$
\begin{aligned}
\sigma_{E}=\frac{1}{2 \pi} & {\left[-c_{3232}^{(0)} \beta i m \int_{0}^{\infty}(G(p)-\bar{G}(p)) \cos \left(p x_{1}\right) d p\right.} \\
& \left.+\gamma \int_{0}^{\infty}(G(p)-\bar{G}(p)) \cos \left(p x_{1}\right) i p d p\right], \\
\sigma_{O}=\frac{1}{2 \pi} & {\left[c_{3232}^{(0)} \beta m \int_{0}^{\infty}(G(p)+\bar{G}(p)) \sin \left(p x_{1}\right) d p\right.} \\
& \left.-\gamma \int_{0}^{\infty}(G(p)+\bar{G}(p)) \sin \left(p x_{1}\right) p d p\right] .
\end{aligned}
$$


To satisfy the requirement that the difference in displacement $\Delta u_{3}$ across $x_{2}=0$ is zero for $\left|x_{1}\right|>a$, the function $G(p)$ in (4.8) is chosen in the form

$$
G(p)=G^{\prime}(p)+i G^{\prime \prime}(p)=\int_{0}^{a} s(t) J_{1}(p t) d t+i \int_{0}^{a} r(t) J_{0}(p t) d t
$$

where $J_{0}$ and $J_{1}$ are Bessel functions and $r(t)$ and $s(t)$ are to be determined.

Hence (4.10) and (4.11) yield

$$
\begin{aligned}
\sigma_{E}=\frac{1}{\pi} & {\left[c_{3232}^{(0)} \beta m \int_{0}^{\infty} \cos \left(p x_{1}\right) d p \int_{0}^{a} r(t) J_{0}(p t) d t\right.} \\
& \left.-\gamma \int_{0}^{\infty} \cos \left(p x_{1}\right) p d p \int_{0}^{a} r(t) J_{0}(p t) d t\right], \\
\sigma_{O}=\frac{1}{\pi} & {\left[c_{3232}^{(0)} \beta m \int_{0}^{\infty} \sin \left(p x_{1}\right) d p \int_{0}^{a} s(t) J_{1}(p t) d t\right.} \\
& \left.-\gamma \int_{0}^{\infty} \sin \left(p x_{1}\right) p d p \int_{0}^{a} s(t) J_{1}(p t) d t\right] .
\end{aligned}
$$

Equations (4.13) and (4.14) may be written in the form

$$
\begin{aligned}
\sigma_{E}=\frac{1}{\pi} & {\left[c_{3232}^{(0)} \beta m \int_{0}^{\infty} \cos \left(p x_{1}\right) d p \int_{0}^{a} r(t) J_{0}(p t) d t\right.} \\
& \left.-\gamma \frac{d}{d x_{1}} \int_{0}^{\infty} \sin \left(p x_{1}\right) d p \int_{0}^{a} r(t) J_{0}(p t) d t\right], \\
\sigma_{O}=\frac{1}{\pi} & {\left[c_{3232}^{(0)} \beta m \int_{0}^{\infty} \sin \left(p x_{1}\right) d p \int_{0}^{a} s(t) J_{1}(p t) d t\right.} \\
& \left.+\gamma \frac{d}{d x_{1}} \int_{0}^{\infty} \cos \left(p x_{1}\right) d p \int_{0}^{a} s(t) J_{1}(p t) d t\right] .
\end{aligned}
$$

Interchanging the order of integration in (4.15) and (4.16) yields

$$
\begin{aligned}
\sigma_{E}=\frac{1}{\pi} & {\left[c_{3232}^{(0)} \beta m \int_{0}^{a} r(t) d t \int_{0}^{\infty} \cos \left(p x_{1}\right) J_{0}(p t) d p\right.} \\
& \left.-\gamma \frac{d}{d x_{1}} \int_{0}^{a} r(t) d t \int_{0}^{\infty} \sin \left(p x_{1}\right) J_{0}(p t) d p\right], \\
\sigma_{O}=\frac{1}{\pi} & {\left[c_{3232}^{(0)} \beta m \int_{0}^{a} s(t) d t \int_{0}^{\infty} \sin \left(p x_{1}\right) J_{1}(p t) d p\right.} \\
& \left.+\gamma \frac{d}{d x_{1}} \int_{0}^{a} s(t) d t \int_{0}^{\infty} \cos \left(p x_{1}\right) J_{1}(p t) d p\right] .
\end{aligned}
$$


Now using the results

$$
\begin{aligned}
& \int_{0}^{\infty} \cos (p x) J_{0}(p t) d p= \begin{cases}\left(t^{2}-x^{2}\right)^{-1 / 2} & \text { for } 0<x<t \\
0 & \text { for } t<x<\infty,\end{cases} \\
& \int_{0}^{\infty} \sin (p x) J_{0}(p t) d p= \begin{cases}0 & \text { for } 0<x<t \\
\left(x^{2}-t^{2}\right)^{-1 / 2} & \text { for } t<x<\infty,\end{cases} \\
& \int_{0}^{\infty} \sin (p x) J_{1}(p t) d p= \begin{cases}x t^{-1}\left(t^{2}-x^{2}\right)^{-1 / 2} & \text { for } 0<x<t \\
0 & \text { for } t<x<\infty,\end{cases} \\
& \int_{0}^{\infty} \cos (p x) J_{1}(p t) d p= \begin{cases}t^{-1} & \text { for } 0<x<t \\
-x t^{-1}\left(x^{2}-t^{2}\right)^{-1 / 2}+t^{-1} & \text { for } t<x<\infty,\end{cases}
\end{aligned}
$$

it follows that

$$
\begin{aligned}
\sigma_{E}=\frac{1}{\pi} & {\left[c_{3232}^{(0)} \beta m \int_{x_{1}}^{a} \frac{r(t) d t}{\left(t^{2}-x_{1}^{2}\right)^{1 / 2}}\right.} \\
& \left.-\gamma \frac{d}{d x_{1}} \int_{0}^{x_{1}} \frac{r(t) d t}{\left(x_{1}^{2}-t^{2}\right)^{1 / 2}}\right] \text { for } 0<x_{1}<a, \\
\sigma_{E}= & \frac{1}{\pi}\left[-\gamma \frac{d}{d x_{1}} \int_{0}^{a} \frac{r(t) d t}{\left(x_{1}^{2}-t^{2}\right)^{1 / 2}}\right] \text { for } x_{1}>a, \\
\sigma_{O}=\frac{1}{\pi} & {\left[c_{3232}^{(0)} \beta m \int_{x_{1}}^{a} \frac{s(t) x_{1} d t}{t\left(t^{2}-x_{1}^{2}\right)^{1 / 2}}\right.} \\
& \left.-\frac{\gamma}{x_{1}} \frac{d}{d x_{1}} \int_{0}^{x_{1}} \frac{t s(t) d t}{\left(x_{1}^{2}-t^{2}\right)^{1 / 2}}\right] \text { for } 0<x_{1}<a, \\
\sigma_{O}= & \frac{1}{\pi}\left[-\frac{\gamma}{x_{1}} \frac{d}{d x_{1}} \int_{0}^{a} \frac{t s(t) d t}{\left(x_{1}^{2}-t^{2}\right)^{1 / 2}}\right] \text { for } x_{1}>a .
\end{aligned}
$$

If, on $x_{2}=0$,

$$
\sigma_{32}\left(x_{1}, 0\right)=p\left(x_{1}\right) \quad \text { for }\left|x_{1}\right|<a,
$$

then (4.17) and (4.19) yield

$$
\begin{aligned}
\frac{p\left(x_{1}\right)+p\left(-x_{1}\right)}{2}=\frac{1}{\pi} & {\left[c_{3232}^{(0)} \beta m \int_{x_{1}}^{a} \frac{r(t) d t}{\left(t^{2}-x_{1}^{2}\right)^{1 / 2}}\right.} \\
& \left.-\gamma \frac{d}{d x_{1}} \int_{0}^{x_{1}} \frac{r(t) d t}{\left(x_{1}^{2}-t^{2}\right)^{1 / 2}}\right] \text { for } 0<x_{1}<a, \\
\frac{p\left(x_{1}\right)-p\left(-x_{1}\right)}{2}=\frac{1}{\pi} & {\left[c_{3232}^{(0)} \beta m \int_{x_{1}}^{a} \frac{s(t) x_{1} d t}{t\left(t^{2}-x_{1}^{2}\right)^{1 / 2}}\right.} \\
& \left.-\frac{\gamma}{x_{1}} \frac{d}{d x_{1}} \int_{0}^{x_{1}} \frac{t s(t) d t}{\left(x_{1}^{2}-t^{2}\right)^{1 / 2}}\right] \text { for } 0<x_{1}<a .
\end{aligned}
$$


Equations (4.21) and (4.22) are of the Abel type and may be inverted to yield

$$
\begin{aligned}
& r(t)= \frac{c_{3232}^{(0)} \beta m}{\gamma} \frac{2 t}{\pi} \int_{0}^{t} \frac{d u}{\left(t^{2}-u^{2}\right)^{1 / 2}} \int_{u}^{a} \frac{r(q) d q}{\left(q^{2}-u^{2}\right)^{1 / 2}} \\
&-\frac{t}{\gamma} \int_{-t}^{t} \frac{p(u) d u}{\left(t^{2}-u^{2}\right)^{1 / 2}} \quad \text { for } 0<t<a, \\
& s(t)= \frac{c_{3232}^{(0)} \beta m}{\gamma} \frac{2}{\pi} \int_{0}^{t} \frac{u^{2} d u}{\left(t^{2}-u^{2}\right)^{1 / 2}} \int_{u}^{a} \frac{s(q) d q}{q\left(q^{2}-u^{2}\right)^{1 / 2}} \\
&-\frac{1}{\gamma} \int_{-t}^{t} \frac{u p(u) d u}{\left(t^{2}-u^{2}\right)^{1 / 2}} \quad \text { for } 0<t<a .
\end{aligned}
$$

Provided the ratio $\beta / \gamma$ is sufficiently small, the integral equations (4.23) and (4.24) are suitable for solution by iteration. If

$$
p\left(x_{1}\right)=-p_{0}-x_{1} p_{1}
$$

where $p_{0}$ and $p_{1}$ are constants, then the first approximations to $r(t)$ and $s(t)$ are given by

$$
\begin{aligned}
& r(t)=-\frac{t}{\gamma} \int_{-t}^{t} \frac{p(u) d u}{\left(t^{2}-u^{2}\right)^{1 / 2}}=\frac{\pi p_{0} t}{\gamma} \quad \text { for } 0<t<a, \\
& s(t)=-\frac{1}{\gamma} \int_{-t}^{t} \frac{u p(u) d u}{\left(t^{2}-u^{2}\right)^{1 / 2}}=\frac{\pi p_{1} t^{2}}{2 \gamma} \quad \text { for } 0<t<a,
\end{aligned}
$$

while the first iteration yields

$$
\begin{aligned}
& r(t)=\frac{\pi p_{0} t}{\gamma}+\frac{2 p_{0} c_{3232}^{(0)} \beta m}{\gamma^{2}} t \int_{0}^{\pi / 2}\left(a^{2}-t^{2} \sin ^{2} \theta\right)^{1 / 2} d \theta \quad \text { for } 0<t<a, \\
& s(t)=\frac{\pi p_{1} t^{2}}{2 \gamma}+\frac{p_{1} c_{3232}^{(0)} \beta m}{2 \gamma^{2}} t^{2} \int_{0}^{\pi / 2} \sin ^{2} \theta\left(a^{2}-t^{2} \sin ^{2} \theta\right)^{1 / 2} d \theta \quad \text { for } 0<t<a .
\end{aligned}
$$

\section{Crack tip stress intensity factors}

The stress $\sigma_{32}$ near the crack tip on $x_{2}=0$ for $x_{2}>a$ may be obtained from (4.18) and (4.20) in the form

$$
\sigma_{32}\left(x_{1}, 0\right)=-\frac{\gamma}{\pi} \frac{d}{d x_{1}} \int_{0}^{a} \frac{r(t) d t}{\left(x_{1}^{2}-t^{2}\right)^{1 / 2}}-\frac{\gamma}{\pi x_{1}} \frac{d}{d x_{1}} \int_{0}^{a} \frac{t s(t) d t}{\left(x_{1}^{2}-t^{2}\right)^{1 / 2}} \quad \text { for } x_{1}>a .
$$

Integration by parts yields the stress intensity factor $\mathcal{K}$ :

$$
\mathcal{K}=\lim _{x_{1} \rightarrow a+}\left(x_{1}-a\right)^{1 / 2} \sigma_{32}\left(x_{1}, 0\right)=\frac{\gamma[r(a)+s(a)]}{\pi(2 a)^{1 / 2}} .
$$


For $\beta / \gamma$ sufficiently small, (5.1) together with (4.25) provides an approximate stress intensity factor $\mathcal{K}_{a}$ in the form

$$
\mathcal{K}_{a}=p_{0}\left(\frac{a}{2}\right)^{1 / 2}\left[1+\frac{2 c_{3232}^{(0)} \beta m a}{\gamma \pi}\right]+p_{1}\left(\frac{a}{2}\right)^{3 / 2}\left[1+\frac{c_{3232}^{(0)} \beta m a}{3 \gamma \pi}\right] .
$$

Now since $m$ denotes the imaginary part of $M$, it follows from (3.14) and (3.17) that

$$
m=-\left[c_{3232}^{(0)} c_{3131}^{(0)}-\left(c_{3231}^{(0)}\right)^{2}\right]^{-1 / 2} .
$$

Use of (5.3) in (5.2) yields

$$
\begin{aligned}
\mathcal{K}_{a}=p_{0} & \left(\frac{a}{2}\right)^{1 / 2}\left[1-\frac{2 c_{3232}^{(0)} \beta a}{\gamma \pi\left[c_{3232}^{(0)} c_{3131}^{(0)}-\left(c_{3231}^{(0)}\right)^{2}\right]^{1 / 2}}\right] \\
& +p_{1}\left(\frac{a}{2}\right)^{3 / 2}\left[1-\frac{c_{3232}^{(0)} \beta a}{3 \gamma \pi\left[c_{3232}^{(0)} c_{3131}^{(0)}-\left(c_{3231}^{(0)}\right)^{2}\right]^{1 / 2}}\right] .
\end{aligned}
$$

For an isotropic material, $c_{3231}=0$ and $c_{3232}=c_{3131}=\mu=\mu^{(0)}\left(\beta\left|x_{2}\right|+\gamma\right)^{2}$, where $\mu$ is the shear modulus. In this case $c_{3232}^{(0)}=c_{3131}^{(0)}=\mu^{(0)}$ and $c_{3231}^{(0)}=0$, and hence (5.4) provides the approximate stress intensity factor in the form

$$
\mathcal{K}_{a}=p_{0}\left(\frac{a}{2}\right)^{1 / 2}\left[1-\frac{2 \beta a}{\gamma \pi}\right]+p_{1}\left(\frac{a}{2}\right)^{3 / 2}\left[1-\frac{\beta a}{3 \gamma \pi}\right] .
$$

Formula (5.4) facilitates an examination of the effect of anisotropy and the inhomogeneity on the crack tip stress intensity factor. For a fixed $c_{3232}^{(0)}$ and $c_{3131}^{(0)}$, an increase in the modulus $c_{3231}^{(0)}$ causes a decrease in the stress intensity factor. For a fixed positive $c_{3232}^{(0)}$ and a fixed $c_{3231}^{(0)} \geq 0$, an increase in the modulus $c_{3131}^{(0)}$ causes an increase in the stress intensity factor. Also, for fixed $c_{3232}^{(0)}, c_{3131}^{(0)}$ and $c_{3231}^{(0)}$, an increase in the ratio $\beta / \gamma$ causes a decrease in the stress intensity factor. Thus, for a fixed $\gamma$, an increase in the parameter $\beta$ in the inhomogeneous modulus causes a reduction in the stress intensity factor.

\section{The displacement over the crack faces}

From (4.4), (4.7) and (4.12) the displacement on the crack faces is given by

$$
\begin{aligned}
u_{3}\left(x_{1}, 0-\right)= & \frac{1}{\pi \gamma} \Re\left[i m \int_{0}^{\infty} \bar{G}(p) \exp \left(-i p x_{1}\right) d p\right] \\
= & \frac{1}{\pi \gamma} \Re i m\left[\int_{0}^{\infty} \exp \left(-i p x_{1}\right) d p \int_{0}^{a} s(t) J_{1}(p t) d t\right. \\
& \left.\quad-i \int_{0}^{\infty} \exp \left(-i p x_{1}\right) d p \int_{0}^{a} r(t) J_{0}(p t) d t\right]
\end{aligned}
$$




$$
\begin{aligned}
& =\frac{m}{\pi \gamma}\left[\int_{0}^{a} s(t) d t \int_{0}^{\infty} \sin \left(p x_{1}\right) J_{1}(p t) d p\right. \\
& \left.\quad+\int_{0}^{a} r(t) d t \int_{0}^{\infty} \cos \left(p x_{1}\right) J_{0}(p t) d p\right] \\
& =\frac{m}{\pi \gamma}\left[\int_{x_{1}}^{a} \frac{x_{1} s(t)}{t\left(t^{2}-x_{1}^{2}\right)^{1 / 2}} d t+\int_{x_{1}}^{a} \frac{r(t) d t}{\left(t^{2}-x_{1}^{2}\right)^{1 / 2}}\right] \\
& \quad \text { for } 0<x_{1}<a,
\end{aligned}
$$

and similarly, from (4.3), (4.7) and (4.12),

$$
\begin{aligned}
u_{3}\left(x_{1}, 0+\right) & =\frac{1}{\pi \gamma} \Re\left[i m \int_{0}^{\infty} G(p) \exp \left(i p x_{1}\right) d p\right] \\
& =-\frac{m}{\pi \gamma}\left[\int_{x_{1}}^{a} \frac{x_{1} s(t)}{t\left(t^{2}-x_{1}^{2}\right)^{1 / 2}} d t+\int_{x_{1}}^{a} \frac{r(t) d t}{\left(t^{2}-x_{1}^{2}\right)^{1 / 2}}\right] \\
& =-u_{3}\left(x_{1}, 0-\right) \text { for } 0<x_{1}<a,
\end{aligned}
$$

where

$$
\lim _{x_{2} \rightarrow 0+} u_{3}\left(x_{1}, x_{2}\right)=u_{3}\left(x_{1}, 0+\right), \quad \lim _{x_{2} \rightarrow 0-} u_{3}\left(x_{1}, x_{2}\right)=u_{3}\left(x_{1}, 0-\right) .
$$

Substitution of (4.25) in (6.2) provides an approximate expression for the crack displacement in the form

$$
\begin{aligned}
u_{3}\left(x_{1}, 0+\right) & \\
= & -\frac{m}{\pi \gamma}\left[\frac{x_{1} \pi p_{1}}{2 \gamma}\left(a^{2}-x_{1}{ }^{2}\right)^{1 / 2}+\frac{\pi p_{0}}{\gamma}\left(a^{2}-x_{1}{ }^{2}\right)^{1 / 2}\right. \\
& +\frac{x_{1} c_{3232}^{(0)} \beta m p_{1}}{2 \gamma^{2}} \int_{x_{1}}^{a} \frac{t}{\left(t^{2}-x_{1}\right)^{1 / 2}} d t \int_{0}^{\pi / 2} \sin ^{2} \theta\left(a^{2}-t^{2} \sin ^{2} \theta\right)^{1 / 2} d \theta \\
& \left.+\frac{2 c_{3232}^{(0)} \beta m p_{0}}{\gamma^{2}} \int_{x_{1}}^{a} \frac{t}{\left(t^{2}-x_{1}^{2}\right)^{1 / 2}} d t \int_{0}^{\pi / 2}\left(a^{2}-t^{2} \sin ^{2} \theta\right)^{1 / 2} d \theta\right] .
\end{aligned}
$$

In particular, the displacement in the middle of the crack where $x_{1}=0$ is

$$
\begin{aligned}
u_{3}(0,0+) & =-\frac{m p_{0}}{\gamma^{2}}\left[a+\frac{2 c_{3232}^{(0)} m \beta}{\pi \gamma} \int_{0}^{a} d t \int_{0}^{\pi / 2}\left(a^{2}-t^{2} \sin ^{2} \theta\right)^{1 / 2} d \theta\right] \\
& =-\frac{m p_{0}}{\gamma^{2}}\left[a+\frac{2 c_{3232}^{(0)} m \beta}{\pi \gamma} \int_{0}^{\pi / 2} d \theta \int_{0}^{a}\left(a^{2}-t^{2} \sin ^{2} \theta\right)^{1 / 2} d t\right] \\
& =-\frac{m p_{0} a}{\gamma^{2}}\left[1+\frac{c_{3232}^{(0)} m \beta a}{\pi \gamma} \int_{0}^{\pi / 2}\left(\cos \theta+\frac{\theta}{\sin \theta}\right) d \theta\right] \\
& =-\frac{m p_{0} a}{\gamma^{2}}\left[1+\frac{c_{3232}^{(0)} m \beta a}{\pi \gamma} I\right],
\end{aligned}
$$


where

$$
I=1+\int_{0}^{\pi / 2} \frac{\theta}{\sin \theta} d \theta \simeq 2.83206 .
$$

Use of (5.3) to substitute for $m$ in (6.4) yields

$$
\begin{aligned}
u_{3}(0,0+)=\frac{p_{0} a}{\gamma^{2}\left[c_{3232}^{(0)} c_{3131}^{(0)}-\left(c_{3231}^{(0)}\right)^{2}\right]^{1 / 2}} \\
\quad \times\left[1-\frac{c_{3232}^{(0)} \beta a I}{\pi \gamma\left[c_{3232}^{(0)} c_{3131}^{(0)}-\left(c_{3231}^{(0)}\right)^{2}\right]^{1 / 2}}\right] .
\end{aligned}
$$

In the isotropic case this reduces to

$$
u_{3}(0,0+)=\frac{p_{0} a}{\gamma^{2} \mu^{(0)}}\left[1-\frac{\beta a I}{\pi \gamma}\right] .
$$

Formula (6.5) facilitates an examination of the effect of anisotropy and the inhomogeneity on the crack displacement. In particular, for a fixed $\gamma>0$ and $\beta=0$, a change in any of the constants $c_{3232}^{(0)}, c_{3131}^{(0)}$ and $c_{3231}^{(0)}$ which causes an increase in the term $c_{3232}^{(0)} c_{3131}^{(0)}-\left(c_{3231}^{(0)}\right)^{2}$ will decrease the crack displacement at $x_{1}=0$. In the case of an inhomogeneous material with fixed $\gamma, c_{3232}^{(0)}, c_{3131}^{(0)}$ and $c_{3231}^{(0)}$, an increase in $\beta / \gamma$ causes a reduction in the crack displacement at $x_{1}=0$.

\section{Numerical results}

The analysis of the previous sections is used to obtain some numerical results for a crack in a functionally graded transversely isotropic material with the elastic moduli varying according to (4.1). For such materials, the $c_{i j k l}^{(0)}$ in (4.1) may be conveniently expressed in terms of five constants $A, N, F, C$ and $L$ (see Clements [6]). If the $x_{3}$ axis is normal to the transverse planes then the nonzero $c_{i j k l}^{(0)}$ are related to the constants $A, N, F, C$ and $L$ by the equations

$$
\begin{array}{lll}
c_{1111}^{(0)}=c_{2222}^{(0)}=A, & c_{1122}^{(0)}=N, & c_{1133}^{(0)}=c_{2233}^{(0)}=F, \\
c_{1313}^{(0)}=c_{2323}^{(0)}=L, & c_{1212}^{(0)}=\frac{A-N}{2}, & c_{3333}^{(0)}=C .
\end{array}
$$

In this case, the $c_{i j k l}^{(0)}$ of interest in the antiplane crack problem are given in terms of the constants $A, N, F, C$ and $L$ by the equations

$$
c_{1313}^{(0)}=c_{2323}^{(0)}=L, \quad c_{1323}^{(0)}=0 .
$$

Expressions for the $c_{i j k l}^{(0)}$ referred to any Cartesian frame of reference in terms of the five constants $A, N, F, C$ and $L$ may be readily obtained from (7.1) by employing the transformation law for fourth-order Cartesian tensors (see Clements [6]). Thus, if the orientation of the Cartesian coordinate frame within the material is such that the $x_{2}$ 
axis is normal to the transverse plane, then the constants $c_{1313}^{(0)}, c_{2323}^{(0)}$ and $c_{1323}^{(0)}$ are given in terms of the constants $A, N, F, C$ and $L$ by the equations

$$
c_{1313}^{(0)}=\frac{A-N}{2}, \quad c_{2323}^{(0)}=L, \quad c_{1323}^{(0)}=0 .
$$

If the $x_{3}$ axis lies in the transverse plane and the $x_{1}$ and $x_{2}$ axes are at an angle of $\pi / 4$ to the transverse plane, then the equations relating the $c_{3 j 3 l}^{(0)}$ to the constants $A, N, F$, $C$ and $L$ take the form

$$
c_{1313}^{(0)}=c_{3232}^{(0)}=\frac{1}{2}\left[\frac{A-N}{2}+L\right], \quad c_{3132}^{(0)}=\frac{1}{2}\left[\frac{A-N}{2}-L\right] .
$$

It is convenient at this point to introduce the nondimensional variables

$$
\begin{gathered}
x=\frac{x_{1}}{a}, \quad C_{i j k l}=\frac{c_{i j k l}^{(0)}}{\mathcal{C}}, \quad P_{0}=\frac{p_{0}}{\mathcal{C}}, \quad P_{1}=\frac{a p_{1}}{\mathcal{C}}, \\
\beta^{\prime}=\beta a, \quad u_{3}^{\prime}=\frac{u_{3}}{a} \quad \text { and } \quad \mathcal{K}_{a}^{\prime}=\frac{\mathcal{K}_{a}}{\sqrt{a} \mathcal{C}},
\end{gathered}
$$

where $\mathcal{C}$ is a reference stress.

In nondimensional form, equations (6.5) for the displacement in the middle of the crack and (5.4) for the stress intensity factor become

$$
\begin{aligned}
\frac{u_{3}^{\prime}(0,0+)}{P_{0}}= & \frac{1}{\gamma^{2}\left[C_{3232} C_{3131}-C_{3231}^{2}\right]^{1 / 2}} \\
& \times\left[1-\frac{C_{3232} \beta^{\prime} I}{\pi \gamma\left[C_{3232} C_{3131}-C_{3231}^{2}\right]^{1 / 2}}\right], \\
\frac{\mathcal{K}_{a}^{\prime}}{P_{0}}= & \frac{1}{\sqrt{2}}\left[1-\frac{2 C_{3232} \beta^{\prime}}{\gamma \pi\left[C_{3232} C_{3131}-C_{3231}^{2}\right]^{1 / 2}}\right] \\
& +\frac{P_{1}}{2 \sqrt{2} P_{0}}\left[1-\frac{C_{3232}^{(0)} \beta^{\prime}}{3 \gamma \pi\left[C_{3232} C_{3131}-C_{3231}^{2}\right]^{1 / 2}}\right] .
\end{aligned}
$$

For the purposes of obtaining numerical values for the stress intensity factor and the crack displacement, sample values of the material constants $A, N, F, C$ and $L$ are chosen to be $A / \mathcal{C}=16.5, N / \mathcal{C}=3.1, F / \mathcal{C}=5, C / \mathcal{C}=6.2$ and $L / \mathcal{C}=3.92$. For a suitably defined value of the reference stress $\mathcal{C}$, these are the constants for a crystal of titanium and are used here for illustrative purposes.

Hence, if the $x_{3}$ axis is normal to the transverse plane, then

$$
C_{1313}=C_{2323}=\frac{L}{\mathcal{C}}=3.92, \quad C_{1323}=0 .
$$

If the $x_{2}$ axis is normal to the transverse plane then

$$
C_{1313}=\frac{A-N}{2 \mathcal{C}}=6.7, \quad C_{2323}=\frac{L}{\mathcal{C}}=3.92, \quad C_{1323}=0 .
$$


TABLE 1 . The values of $\mathcal{K}_{a}^{\prime} / P_{0}$ for $\gamma=1, C_{3131}=3.92, C_{3232}=3.92, C_{3132}=0$ with various values of $\beta^{\prime}$ and $P_{1} / P_{0}$.

\begin{tabular}{llll}
\hline$\beta^{\prime}$ & $\mathcal{K}_{a}^{\prime} / P_{0}$ & $\mathcal{K}_{a}^{\prime} / P_{0}$ & $\mathcal{K}_{a}^{\prime} / P_{0}$ \\
& $P_{1} / P_{0}=0$ & $P_{1} / P_{0}=0.5$ & $P_{1} / P_{0}=1$ \\
\hline 0 & 0.707 & 0.884 & 1.061 \\
0.02 & 0.698 & 0.875 & 1.051 \\
0.04 & 0.689 & 0.865 & 1.041 \\
0.06 & 0.680 & 0.856 & 1.031 \\
0.08 & 0.671 & 0.846 & 1.022 \\
0.10 & 0.662 & 0.837 & 1.012 \\
\hline
\end{tabular}

TABLE 2. The values of $\mathcal{K}_{a}^{\prime} / P_{0}$ for $\gamma=1, C_{3131}=6.7, C_{3232}=3.92, C_{3132}=0$ with various values of $\beta^{\prime}$ and $P_{1} / P_{0}$.

\begin{tabular}{llll}
\hline$\beta^{\prime}$ & $\mathcal{K}_{a}^{\prime} / P_{0}$ & $\mathcal{K}_{a}^{\prime} / P_{0}$ & $\mathcal{K}_{a}^{\prime} / P_{0}$ \\
& $P_{1} / P_{0}=0$ & $P_{1} / P_{0}=0.5$ & $P_{1} / P_{0}=1$ \\
\hline 0 & 0.707 & 0.884 & 1.061 \\
0.02 & 0.700 & 0.877 & 1.053 \\
0.04 & 0.693 & 0.870 & 1.046 \\
0.06 & 0.686 & 0.862 & 1.038 \\
0.08 & 0.680 & 0.855 & 1.031 \\
0.10 & 0.673 & 0.848 & 1.023 \\
\hline
\end{tabular}

If the $x_{3}$ axis lies in the transverse plane and the $x_{1}$ and $x_{2}$ axes are at an angle of $\pi / 4$ to the transverse plane, then

$$
\begin{aligned}
C_{1313}=C_{3232} & =\frac{1}{2 \mathcal{C}}\left[\frac{A-N}{2}+L\right]=5.31 \\
C_{3132} & =\frac{1}{2 \mathcal{C}}\left[\frac{A-N}{2}-L\right]=1.39 .
\end{aligned}
$$

In Tables 1, 2 and 3 and Figure 2, numerical results obtained using (7.2) and (7.3) and the sample material constants (7.4)-(7.7) provide some quantitative information regarding the effect of anisotropy and inhomogeneity on the stress intensity factor and the crack displacement. The values of the stress intensity factors in the tables are correct to three decimal places.

Tables 1, 2 and 3 illustrate that for fixed values of the constants $C_{3 j 3 l}$ and $\gamma$, an increase in $\beta^{\prime}$ and thus the gradient of the elastic moduli $c_{3 j 3 l} / \mathcal{C}=C_{3 j 3 l}\left(\beta^{\prime}\left|x_{2}\right|+\gamma\right)^{2}$ causes a decrease in the stress intensity factors.

If the orientation of the Cartesian frame within the sample transversely isotropic material is such that the $x_{3}$ axis is normal to the transverse plane, then the constants $C_{i j k l}$ are given by (7.4), the stress intensity factors are given in Table 1 and the midcrack displacement is given in Figure 2. 


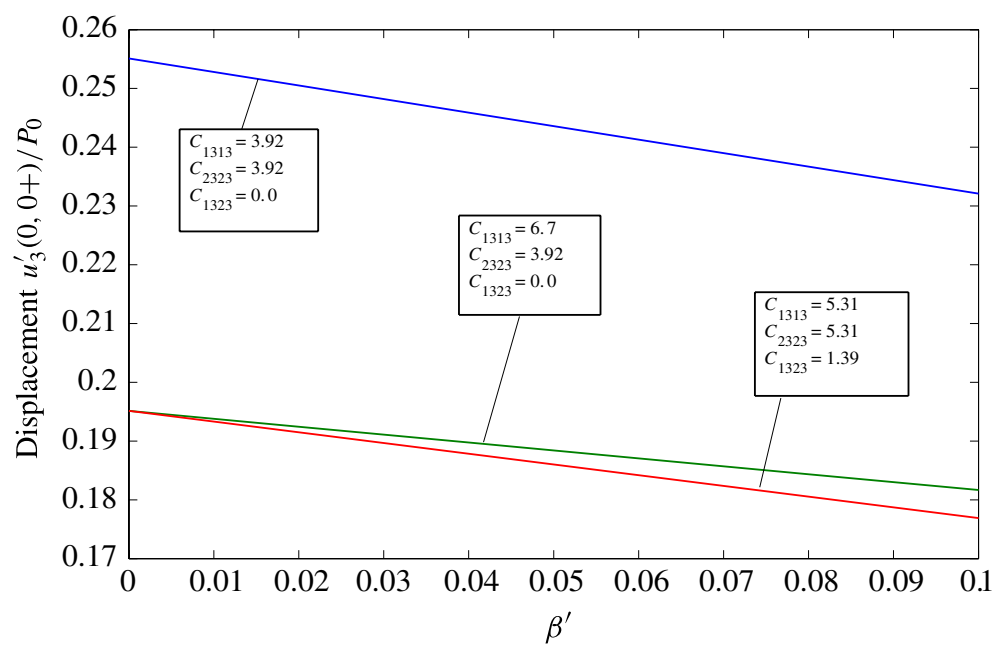

FIGURE 2. Displacement $u_{3}^{\prime}(0,0+) / P_{0}$ for various values of $\beta^{\prime}$.

TABLE 3 . The values of $\mathcal{K}_{a}^{\prime} / P_{0}$ for $\gamma=1, C_{3131}=5.31, C_{3232}=5.31, C_{3132}=1.39$ with various values of $\beta^{\prime}$ and $P_{1} / P_{0}$.

\begin{tabular}{llll}
\hline$\beta^{\prime}$ & $\mathcal{K}_{a}^{\prime} / P_{0}$ & $\mathcal{K}_{a}^{\prime} / P_{0}$ & $\mathcal{K}_{a}^{\prime} / P_{0}$ \\
& $P_{1} / P_{0}=0$ & $P_{1} / P_{0}=0.5$ & $P_{1} / P_{0}=1$ \\
\hline 0 & 0.707 & 0.884 & 1.061 \\
0.02 & 0.698 & 0.874 & 1.051 \\
0.04 & 0.688 & 0.864 & 1.040 \\
0.06 & 0.679 & 0.855 & 1.030 \\
0.08 & 0.670 & 0.845 & 1.020 \\
0.10 & 0.660 & 0.835 & 1.010 \\
\hline
\end{tabular}

If the Cartesian frame is reoriented within the material so that the $x_{2}$ axis is normal to the transverse plane, then, from (7.4) and (7.5), the constants $C_{3232}=3.92$ and $C_{3231}=0$ remain unchanged while $C_{3131}$ increases from 3.92 to 6.7 . Also $C_{3232} C_{3131}-C_{3231}^{2}=26.264$. As a result, for a fixed value of $\beta^{\prime}>0$, there is an increase in the stress intensity factor but a substantial decrease in the antiplane displacement at the crack centre (see Figure 2).

If the Cartesian frame is reoriented within the material so that the $x_{3}$ axis lies in the transverse plane and the $x_{1}$ and $x_{2}$ axes are at an angle of $\pi / 4$ to the transverse plane, then, from (7.6) and (7.7), $C_{2323}=C_{3131}=5.31$ and $C_{3231}=1.39$. Note that $C_{3232} C_{3131}-C_{3231}^{2}=26.264$, which is the same as the corresponding value in the previous case, and as a consequence the displacement when $\beta^{\prime}=0$ calculated from (7.2) is identical to the corresponding displacement for the previous orientation of the 
Cartesian frame (see Figure 2). Also from Figure 2, due to the larger value of $C_{3232}$ the displacement for $\beta^{\prime}>0$ is less than the corresponding displacement in the previous case. The stress intensity factors are given in Table 3 . These values are less than the corresponding values in Tables 1 and 2, showing that this orientation of the Cartesian frame within the transversely isotropic material gives rise to lower stress intensity factors than the two previous orientations.

\section{Final remarks}

An antiplane crack problem has been considered for an anisotropic FGM in which the elastic moduli increase quadratically with distance in the direction perpendicular to the plane crack faces. Formulae for the crack tip stress intensity factors and the displacement over the crack faces are given in terms of solutions to Fredholm integral equations. These equations yield an approximate analytical solution for a restricted class of inhomogeneous elastic materials. The solutions obtained provide qualitative and quantitative information regarding the reduction in the stress intensity factors and the crack face displacement as the gradient of the quadratic variation in the elastic moduli increases.

\section{References}

[1] M. I. Azis and D. L. Clements, "A boundary element method for anisotropic inhomogeneous elasticity", Internat. J. Solids Structures 38 (2001) 5747-5764.

[2] H. Bohr, "Cracks in functionally graded materials", Math. Sci. Eng. A 362 (2003) 40-60.

[3] Y.-S. Chan, G. Paulino and A. C. Fanning, "The crack problem for nonhomogeneous materials under antiplane shear loading-a displacement based formulation", Internat. J. Solids Structures 38 (2001) 2989-3005.

[4] Y. F. Chen and F. Erdogan, "The interface crack problem for a nonhomogeneous coating bonded to a homogeneous substrate", J. Mech. Phys. Solids 44 (1996) 771-787.

[5] Y. Z. Chen, X. Y. Lin and Z. X. Wang, "Antiplane elasticity crack problem for a strip of functionally graded materials with mixed boundary condition", Mech. Res. Comm. 37 (2010) 50-53.

[6] D. L. Clements, Boundary value problems governed by second order elliptic systems (Pitman, Bath, 1981).

[7] D. L. Clements and W. T. Ang, "On a generalised plane strain crack problem for inhomogeneous anisotropic elastic materials", Internat. J. Engrg. Sci. 44 (2006) 273-284.

[8] D. L. Clements, C. Atkinson and C. Rogers, "Antiplane crack problems for an inhomogeneous elastic material", Acta Mech. 29 (1978) 199-211.

[9] S. Dag and F. Erdogan, "A surface crack in a graded medium loaded by a sliding rigid stamp", Eng. Fract. Mech. 69 (2002) 1729-1751.

[10] F. Erdogan, "The crack problem for bonded nonhomogeneous materials under antiplane shear loading", J. Appl. Mech. Trans. ASME 52 (1985) 823-828.

[11] F. Erdogan and M. Ozturk, "The interface crack problem for a nonhomogeneous coating bonded to a homogeneous substrate", Internat. J. Engrg. Sci. 30 (1992) 1507-1523.

[12] J. D. Eshelby, W. T. Read and W. Shockley, "Anisotropic elasticity with applications to dislocation theory", Acta Metall. 1 (1953) 251-259.

[13] Y. Gelbstein, M. P. Daniel and Z. Dashevsky, "Powder metallurgical processing of functionally graded p- $\mathrm{Pb}_{1-x} \mathrm{Sn}_{x}$ Te materials for thermoelectric applications", Phys. B: Condensed Matter 391 (2007) 256-265. 
[14] H. Hassanin and K. Jiang, "Functionally graded microceramic components", Microelectron. Eng. 87 (2010) 1610-1613.

[15] Z.-H. Jin and R. C. Batra, "Some basic fracture mechanics concepts in functionally graded materials", J. Mech. Phys. Solids 44 (1996) 1221-1235.

[16] N. Konda and F. Erdogan, "The mixed mode crack problem in a nonhomogeneous elastic medium", Eng. Fract. Mech. (1994) 533-545.

[17] N. Noda, "Thermal stress in functionally graded materials", J. Thermal Stresses 22 (1999) $477-512$.

[18] N. Noda and B. L. Wang, "Transient thermoelastic responses of functionally graded materials containing collinear cracks", Eng. Fract. Mech. 69 (2002) 1791-1809.

[19] G. Paulino, "Fracture of functionally graded materials", Eng. Fract. Mech. 69 (2002) 1519-1520.

[20] W. Pompe, H. Worch, M. Epple, W. Friess, M. Galinsky, P. Greil, U. Hempel, D. Scharnweber and K. Schulte, "Functionally graded materials in biomedical applications", Math. Sci. Eng. A 362 (2003) 40-60.

[21] H. Riedel, B. Kieback and A. Neubrand, "Processing techniques for functionally graded materials", Math. Sci. Eng. A 362 (2003) 81-105.

[22] A. N. Stroh, "Dislocations and cracks in anisotropic elasticity", Philos. Mag. 3 (1958) 625-646.

[23] X. H. Zhang, Y. W. Mai and B. L. Wang, "Functionally graded materials under severe thermal environments", J. Amer. Ceram. Soc. 88 (2005) 683-690. 